\title{
Repair of the left ventricular wall pseudoaneurysm following myocardial infarction (simple modified dor technique)
}

\author{
Mahmood Hosseinzadeh Maleki, Nahid Azdaki, Mohammad Reza Rezvani and Alireza Rahmani Motlaq* \\ Cardiovascular Diseases Research Center, Birjand University of Medical Sciences, Birjand, Iran
}

\begin{abstract}
\section{Introduction}

Rupture of the left ventricular (LV) wall is a rare but disastrous complication of myocardial infarction [1,2]. It is hardly contained by hematoma and pericardial adhesions creating a pseudoaneurysm (PsA) [1]. Urgent surgical repair is required to prevent catastrophic rupture. Surgical repair aims to exclude the necrotic myocardium and aneurysmal area with a patch to preserve the LV geometry and performance [3]. We report a case of LV PsA that was surgically repaired with a modified Dor technique.
\end{abstract}

A rare however serious complication of myocardial infarction is left ventricular pseudoaneurysm that requires urgent intervention to avoid unforeseeable fatal rupture. Here, we present a successful repair of posterolateral and basal left ventricular pseudoaneurysm case, using a modified Dor technique.

\section{Case presentation}

A 74-year old male patient with no significant past medical history was presented with a 6-week history of dyspnea. A chest X-ray was performed to evaluate symptoms that depicted an enlarged cardiac silhouette. This was followed by transthoracic echocardiography that showed huge posterolateral and basal LV pseudoaneurysm $(7 \times 10 \mathrm{~cm})$, LV systolic dysfunction (ejection fraction $(\mathrm{EF}) \leq 20 \%$ ), mild mitral regurgitation (MR) and Aortic insufficiency (AI). Consequently, the patient underwent left heart catheterization that revealed a totally occluded left circumflex coronary artery, $80 \%$ stenotic lesion in the left anterior descending artery, intimal irregularity in the right coronary artery, and large posterobasal and lateral LV pseudoaneurysm.

On the following day, after Intera aortic balloon pump insertion (IABP), the patient underwent surgical repair of LV PsA (Dor procedure) using the modified technique along with bypass grafting of the left coronary system lesions.

Upon opening the pericardium, dense fibrinous adhesions were found suggestive of reactive pericarditis secondary to contained LV rupture. The pericardial adhesions were gently dissected. After initiating cardiopulmonary bypass, the heart was lifted upwards and contained rupture of a large posterolateral LV PsA was known. The posterior wall of LV was opened over the PsA area and an organized thrombus, which was sealing off the rupture site, was removed. The diagnosis of LV PsA was confirmed by the absence of myocardial components in the wall (e.g. thinned necrotic myocardium), formed by organized hematoma and pericardial adhesions. Inside the LV cavity, the mitral valve was intact, while the posterior papillary muscle appeared normal with hyperaemic borders suggestive of recent ischaemic injury. The Dacron patch was prepared. Interrupted 2-0 Etiband-pledged mattress sutures were placed around the neck of the PsA, then passed through the Dacron patch and tied down (Figure 1A and 1B).

Besides, the patient underwent bypass grafting of the left anterior descending artery using the left internal mammary artery. The crossclamp time was 135 minutes, total cardiopulmonary bypass time was 156 minutes, and the total procedure time was 4 hours.

IABP extract after 52 hours. Postoperative echocardiography showed improvement of LV function (EF $\leq 30 \%$ ), mild MR, and mild AI. Also, the Dacron Patch appeared intact with no paradoxical bulging during systole and no flow across (Figure 2). The patient was discharged on the 10th postoperative day.

\section{Discussion}

Patients with LV PsA commonly present with angina or congestive heart failure symptoms but can also present with cardiac tamponade [4].

In this case report, the patient presented with dyspnea, while he had no history of cardiac disease before the time of ischemic injury. However, operative findings were suggestive of a sub-acute presentation organized thrombus and thickened pericardial tissues sealing off the LV rupture site. Besides, the rupture site edges appeared thickened and scarred. Differentiation between true and pseudo-LV aneurysms is crucial for the timing of intervention.

${ }^{\star}$ Correspondence to: Alireza Rahmani Motlaq, Medical student, Birjand University of Medical Sciences, Birjand, Iran, Tel: +98 9215225961; E-mail: alireza.rahmani@bums.ac.ir

Key words: pseudoaneurysm, dor technique, left ventricular geometry

Received: June 17, 2020; Accepted: June 26, 2020; Published: June 30, 2020 


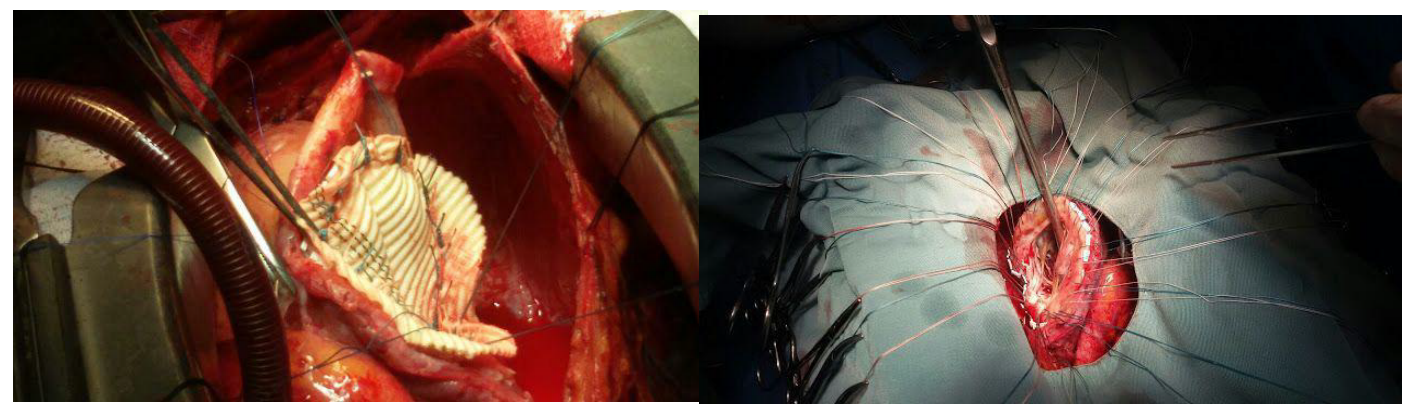

Figure 1. Repair of PsA with the Dacron patch

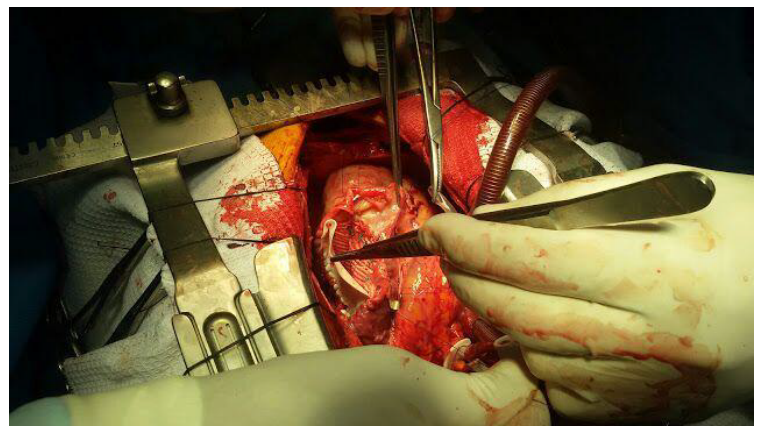

Figure 2. Reinforcement of PsA repair with the Gore-tex patch

The basic principle of the Dor procedure is to exclude the LV aneurysmal part with a circular patch to restore the physiological geometry of the LV cavity and to improve its function $[5,6]$. The two patch techniques may provide an additional benefit over the traditional single-layer patch by being less prone to bulging out during systole; hence, they result in better preservation of the LV geometry and function, especially in the early postoperative period. To our knowledge, this is the first reported case of LV PsA repair using two patches. Long-term follow-up is needed to evaluate the effect of the two patch techniques on LV remodeling.

\section{References}

1. Zoffoli G, Mangino D, Venturini A, Terrini A, Asta A, et al. (2009) Diagnosing left ventricular aneurysm from pseudo-aneurysm: A case report and a review in literature. $J$ Cardiothorac Surg 4:1-5. [Crossref]
2. Hosseinzadeh-Maleki M, Valizadeh N, Rafatpanah N, Moezi SA (2015) Survival after left ventricular free wall rupture due to acute myocardial infarction. ARYA Atheroscler 11: 310-313. [Crossref]

3. Elgharably H, Halbreiner MS, Shoenhagen P, Navia JL (2016) Repair of the left ventricular pseudoaneurysm with the triple patch technique (Empanada Patch). Interact Cardiovasc Thorac Surg 22:116-117. [Crossref]

4. Hulten EA, Blankstein R (2012) Pseudoaneurysms of the heart. Circulation 125:19201925. [Crossref]

5. Buckberg GD, Dor V, Di Donato M, Sabatier M, Montiglio F, et al. (2001) Ventricular shape and function in health and diseaseleft ventricular reconstruction by endoventricular circular patch plasty repair: A 17-year experience. Sem Thor Cardiovas Sur 13: 435-447.

6. Dor V, Saab M, Coste P, Kornaszewska M, Montiglio F (1989) Left ventricular aneurysm: A new surgical approach. Thorac Cardiovasc Surg 37: 11-19. [Crossref]

Copyright: $\odot 2020$ Maleki MH. This is an open-access article distributed under the terms of the Creative Commons Attribution License, which permits unrestricted use, distribution, and reproduction in any medium, provided the original author and source are credited. 